Pacific Journal of Mathematics

ON ALGEBRA ACTIONS ON A GROUP ALGEBRA 


\title{
ON ALGEBRA ACTIONS ON A GROUP ALGEBRA
}

\author{
J. E. KERLIN, JR.
}

A complete description of all algebra actions of the group algebra $L^{1}(K)$ on the group algebra $L^{1}(G)[M(G)]$ for locally compact Abelian groups $K$ and $G$ is presented. A fundamental algebra action of $L^{1}(K)$ on $L^{1}(G)$ is that induced by a continuous homomorphism $\theta: K \rightarrow G$ via a generalized convolution; such actions have been considered by Gelbaum in characterizing topological tensor products of group algebras. It is shown in this paper that conversely every algebra action of $L^{1}(K)$ on $L^{1}(G)[M(G)]$ is induced by a necessarily continuous homomorphism of $K$ into the quotient of $G$ by a compact subgroup. The analysis is based on a representation theorem for algebra actions on $L^{1}(G)$ for general locally compact group $G$. Namely, every algebra action of a Banach algebra $C$ on $L^{1}(G)$ is the composition of a necessarily continuous cetral homomorphism $\Psi$ of $C$ into $M(G)$ and convolution in $M(G)$ : $c \cdot a=\Psi(c) * a$ for all $c \in C$ and $a \in L^{1}(G)$. Applications to topological tensor products of group algebras are announced.

Let $G$ and $K$ be locally compact Abelian groups, and, let $\theta$ be a continuous homomorphism of $K$ into $G$. Gelbaum [3] has observed that $\theta$ induces a module action of the group algebra $L^{1}(K)$ on the group algebra $L^{1}(G)$ via a "generalized convolution": if $c \in L^{1}(K)$ and $a \in L^{1}(G)$, then $c *_{0} a \in L^{1}(G)$ is given by

$$
c *_{\theta} \alpha(g)=\int_{\pi} c(k) a(g-\theta(k)) d k, \quad(g \in G) .
$$

Moreover, this module action is associative in the sense that

$$
\left(c *_{\theta} a\right) * a^{\prime}=c *_{\theta}\left(\alpha * a^{\prime}\right) \quad\left(=\alpha *\left(c *_{\theta} a^{\prime}\right)\right.
$$

by commutativity of $L^{1}(G)$ ), and, the action satisfies the inequality $\left\|c *_{\theta} a\right\|_{1} \leqq\|c\|_{1}\|a\|_{1}$. Hence $L^{1}(G)$ is an algebra over $L^{1}(K)$ and the action is continuous, i.e., $L^{1}(G)$ is an (isometric) Banach $L^{1}(K)$-algebra.

The question we pose is "Does the converse hold, i.e., if $L^{1}(G)$ is an algebra over $L^{1}(K)$ such that $\|c \cdot a\|_{1} \leqq\|c\|_{1}\|a\|_{1}$ for all $c \in L^{1}(K)$ and $a \in L^{1}(G)$, is the action of $L^{1}(K)$ on $L^{1}(G)$ induced by a continuous homomorphism of $K$ into $G$ ?" We answer this question in full, the main result culminating in Corollary 3.2.

I would like to take this opportunity to thank Professor Bernard Gelbaum, who suggested the above problem, for his kind encouragement and stimulating discussions during the preparation of part of this work. The author also thanks the referee for his numerous 
constructive comments and suggestions in an earlier version of this paper.

1. Preliminaries. Let $G$ be a locally compact group. $M(G)$ will denote the Banach algebra of all finite, complex-valued, regular Borel measures on $G$ with convolution as multiplication. The convolution of two measures, $\mu$ and $\nu$, in $M(G)$ will be written as $\mu * \nu . L^{1}(G)$ will denote the Banach algebra of (equivalence classes of) complexvalued measurable functions on $G$ summable with respect to left Haar measure with multiplication given by convolution. We will from time to time also regard $L^{1}(G)$ as the closed two-sided ideal of $M(G)$ consisting of those measures absolutely continuous with respect to (left) Haar measure on $G$; the details of this identification can be found in [5]. Finally, if $G$ is Abelian, then $\hat{G}$ will denote the Pontryagin dual group of $G$, i.e., the group of continuous characters on $G$. If $\alpha \in \widehat{G}$, then the value of $\alpha$ at $g \in G$ will be denoted by $(g, \alpha)$. The Fourier [-Stieltjes] transform of $a \in L^{1}(G)[\mu \in M(G)]$ is given by

$$
\hat{a}(\alpha)=\int_{G} \overline{(g, \alpha)} a(g) d g\left[\hat{\mu}(a)=\int_{G} \overline{(g, \alpha)} d \mu(g)\right]
$$

for all $\alpha \in \hat{G}$.

Definition 1.1. Let $C$ be a Banach algebra. $A$ Banach algebra $A$, is a $C$-algebra if there is a complex bilinear mapping $C \times A \ni(c, a) \rightarrow$ $c \cdot a \in A$ such that

(i ) $\left(c c^{\prime}\right) \cdot a=c \cdot\left(c^{\prime} \cdot a^{\prime}\right)$

(ii) $c \cdot\left(a a^{\prime}\right)=(c \cdot a) a^{\prime}=a\left(c \cdot a^{\prime}\right)$

for all $c, c^{\prime} \in C$ and $a, a^{\prime} \in A$. We call $A$ a Banach $C$-algebra if in addition there is a nonnegative constant $\chi$ such that

(iii) $\|c \cdot a\|_{A} \leqq \chi\|c\|_{C}\|a\|_{A}$

for all $c \in C$ and $a \in A$. We will refer to the least such nonnegative constant $\chi$ satisfying (iii) as the norm of the action of $C$ on $A$ (this "norm" is the bilinear norm of the complex bilinear map $(c, a) \rightarrow c \cdot a)$. If $\chi$ can be chosen to be 0 in (iii), then we say: $A$ is a degenerate $C$-algebra, i.e., $c \cdot a=0$ for all $c \in C$ and $a \in A$. If we can take $\chi=1$ in (iii) then we say $A$ is an isometric Banach $C$-algebra (following [11]). (Note that if $A$ is a commutative Banach algebra, then the last equality in (ii) follows from the first equality in (ii).)

Introductory properties and examples of Banach modules are discussed [6]. Further discussions, relevent to this paper, appear in [3], [4], and [11].

The following example provides a basis for our discussions. Let $K$ and $G$ be (not necessarily Abelian) locally compact groups. Let $\theta: K \rightarrow G$ be a continuous homomorphism. Then $L^{1}(G)$ becomes an isometric Banach $L^{1}(K)$-module via " $\theta$-convolution" in the following 
manner. If $c \in L^{1}(K)$ and $a \in L^{1}(G)$, then $c *_{\theta} a \in L^{1}(G)$ is defined by

$$
c *_{\theta} a(g)=\int_{K} c(k) a\left(\theta(k)^{-1} g\right) d k \quad(g \in G) ;
$$

that is, the right hand side of (1) is finite $d g$-a.e. and defines a $d g$-measurable function and element in $L^{1}(G)$ and is defined independent of the choice of representatives $c(k)$ and $a(g)$ in the equivalence classes (modulo null functions) determined by $c \in L^{1}(K)$ and $a \in L^{1}(G)$. Moreover, we have $\left\|c *_{\theta} a\right\|_{1} \leqq\|c\|_{1}\|a\|_{1}$. This Banach module action of $L^{1}(K)$ on $L^{1}(G)$ becomes an algebra action if and only if $\theta$ is central, i.e., $\theta$ maps $K$ into the center of $G$.

In general now, if $\theta: K \rightarrow G$ is a continuous homomorphism, then $\theta^{*}$ will denote the canonical norm decreasing homomorphism of $L^{1}(K)$ into $M(G)$ such that

$$
\left\langle f, \theta^{*}(c)\right\rangle=\int_{G} f(g) d g^{*}(c)(g)=\int_{K} f(\theta(k)) c(b) d k=\langle f \circ \theta, c\rangle
$$

for all $c \in L^{1}(K)$ and $f \in C_{0}(G)$, the Banach algebra of complex-valued continuous functions vanishing at infinity on $G$ with the sup-norm. It is easy to check that $c *_{\theta} a=\theta^{*}(c) * a, c \in L^{1}(K), a \in L^{1}(G)$, and also that $\theta$ is central if and only if $\theta^{*}$ is central. Finally, if $K$ and $G$ are Abelian and if $\hat{\theta}: \hat{G} \rightarrow \hat{K}$ is the dual homomorphism of $\theta:$ ([5], p. 392) $(k, \hat{\theta}(\alpha))=(\theta(k), \alpha), k \in K, \alpha \in \hat{G}$, then $\left(\theta^{*}(c)\right)^{\wedge}=\hat{c} \circ \hat{\theta}$ for all $c \in L^{1}(K)$.

1. Algebra actions on $\mathrm{L}^{1}(\mathrm{G})$. Let $K$ and $G$ be $L C$ groups. We have observed that a continuous central homomorphism $\theta$ of $K$ into $G$ induces a canonical continuous central homomorphism $\theta^{*}$ of $L^{1}(K)$ into $M(G)$ such that the action $c \cdot a \equiv \theta^{*}(c) * a \quad\left(=c *_{\theta} a\right), c \in L^{1}(K)$, $a \in L^{1}(G)$, makes $L^{1}(G)$ into a Banach $L^{1}(K)$-algebra. More generally, if $\Psi$ is any complex homomorphism of a Banach algebra $C$ into the center of $M(G)$, then the action $c \cdot a \equiv \Psi(c) * a, c \in C, a \in L^{1}(G)$, makes $L^{1}(G)$ into a Banach $C$-algebra. (The striking fact that the module action is necessarily continuous will become clear later.) The main purpose now is to show the converse holds.

If $C$ is a commutative Banach algebra, we denote by $\mathfrak{M}_{C}^{3}$ and $\mathfrak{M}_{C}$ the spaces of all and all nonzero multiplicative linear functionals on $C$, respectively. Give each space the weak*, topology. The Gelfand transform of $c \in C$ is denoted by $\hat{c}: \hat{c}(\phi)=\phi(c), \phi \in \mathbb{M}_{C}^{0}$.

The following is a straightforward generalization of Lemmas 1,2 [3], p. 134.

Proposition 2.1. Let $A$ and $C$ be commutative Banach algebras. Suppose $A$ is a C-algebra. Then there is a continuous map $\mu: \mathfrak{M}_{A} \rightarrow$ 
$\mathfrak{M}_{c}^{0}$ such that

$$
[c \cdot a]^{\wedge}=\hat{c} \circ \mu \hat{a}
$$

for all $c \in C$ and $a \in A$.

We are now prepared to present the main theorem in this section.

THEOREM 2.2. Let $C$ be a Banach algebra and let $G$ be a locally compact group. Suppose $L^{1}(G)$ is a C-algebra. Then the module action is necessarily continuous and there is a continuous unique central homomorphism $\Psi: C \rightarrow M(G)$ such that:

(i ) $c \cdot a=\Psi(c) * a$ for all $c \in C$ and $a \in L^{1}(G)$;

(ii) $\|\Psi\|$ is the norm of the action of $C$ on $L^{1}(G)$.

Finally, if $C$ is commutative and $G$ is abelian, and, if $\mu: \hat{G} \rightarrow \mathfrak{M}_{C}^{0}$ is the adjoint map induced by the action of $C$ on $L^{1}(G)$ (as in Proposition 2.1), then

(iii) $(\Psi c)^{\wedge}=\hat{c} \circ \mu$ on $\hat{G}$ for all $c \in C$.

Proof. (1) For each $c \in C$, define the linear operator $T_{c}$ on $L^{1}(G)$ by $T_{c}(a) \equiv c \cdot a, a \in L^{1}(G)$. Since $L^{1}(G)$ is an algebra over $C$, c.f., condition (ii) in Def. 1.1, $T_{c}$ is a centralizer of $L^{1}(G)$ :

$$
T_{c}\left(a * a^{\prime}\right)=T_{c}(\alpha) * a^{\prime}=a * T_{c}\left(a^{\prime}\right)
$$

for all $a, a^{\prime} \in L^{1}(G)$. It is well known (e.g., [7, Theorem 2.1]) that every centralizer on $L^{1}(G)$ is a bounded linear operator and therefore $T_{c}$ is a bounded linear operator on $L^{1}(G)$. Since $\mathrm{T}_{c}$ is a (right) centralizer, by Wendel [13, Theorem 1] there is a measure $\Psi(c) \in M(G)$ such that $T_{c}(a)=\Psi(c) * a$ for all $a \in L^{1}(G)$; moreover, $\left\|T_{c}\right\|=\|\Psi(c)\|$. Clearly we have $c \cdot a=\Psi(c) * a, c \in L^{1}(K), a \in L^{1}(G)$.

(2) We show $\Psi$ is a central homomorphism, i.e., $\Psi$ is a homomorphism of $C$ into the center of $M(G)$. First, observe that $\Psi(c) * a=$ $a * \Psi(c)$ for all $c \in C$ and $a \in L^{1}(G)$. Indeed, for every $a^{\prime} \in L^{1}(G)$

$$
(\Psi(c) * a) * a^{\prime}=(c \cdot a) * a^{\prime}=a *\left(c \cdot a^{\prime}\right)=a *\left(\Psi(c) * a^{\prime}\right)=(a * \Psi(c)) * a^{\prime}
$$

and hence $\Psi(c) * a=a * \Psi(c)$ since $L^{1}(G)$ has a right approximate identity. Using the fact that $L^{1}(G)$ is an ideal in $M(G)$ and $\Psi c$ commutes with $L^{1}(G)$, we have

$$
\begin{aligned}
a *(\nu * \Psi(c)) & =(a * \nu) * \Psi(c)=\Psi(c) *(a * \nu) \\
& =(\Psi(c) * a) * \nu=(a * \Psi(c)) * \nu \\
& =a *(\Psi(c) * \nu)
\end{aligned}
$$

for all $a \in L^{\mathrm{l}}(G)$, i.e., the mapping $a \rightarrow a *\left(\nu * \Psi(c)-\Psi_{c} * \nu\right)$ is the zero operator on $L^{1}(G)$. By Wendel [13, Theorem 1], the norm of this 
operator is $\|\nu * \Psi(c)-\Psi(c) * \nu\|$, which consequently is 0 . Thus,

$$
\nu * \Psi(c)=\Psi(c) * \nu
$$

for all $\nu \in M(G)$ and $c \in C$, and, $\Psi$ is central. Finally, to show $\Psi$ is a homomorphism we again use Wendel's result that the norm of the operator $a \rightarrow \mu * a$ on $L^{1}(G)$ for $\mu \in M(G)$ is $\|\mu\|$, e.g., if $c, c^{\prime} \in C$, then

$$
\Psi\left(c c^{\prime}\right) * a=\left(c c^{\prime}\right) \cdot a=c \cdot\left(c^{\prime} \cdot a\right)=\Psi(c) * \Psi\left(c^{\prime}\right) * a
$$

for all $a \in L^{1}(G)$ and hence $\left\|\Psi\left(c c^{\prime}\right)-\Psi(c) * \Psi\left(c^{\prime}\right)\right\|=0$, i.e. $\Psi$ is multiplicative; the linearity of $\Psi$ can be similarly shown.

(3) To show $\Psi$ is continuous and $\|\Psi\|$ is identical to the norm $\chi$ of the action of $C$ on $L^{1}(G)$, we first need the following lemma.

Lemma 2.3. Let $G$ be a locally compact group. Then the center of $M(G)$ is a semisimple commutative Banach algebra.

Proof. It is clear that the center of $M(G)$ is a commutative Banach algebra. To see that it is semisimple, consider the left regular representation $\mathscr{L}$ of $M(G)$ on the Hilbert space $L^{2}(G): \mathscr{L}_{\mu}(a)=\mu * a$, $a \in L^{2}(G), \mu \in M(G)$. It is well known that $\mathscr{L}$ is a faithful *-representation of $M(G)$ into the algebra of all bounded linear operators on $L^{2}(G)$. Consequently, $M(G)$ is an $A^{*}$-algebra and hence any *-subalgebra of $M(G)$ is semisimple [10, Theorem 4.1.19]. In particular, the center of $M(G)$ must be semisimple.

Returning to the proof of Theorem 2.2, the complex homomorphism $\Psi$ from $C$ into the center of $M(G)$ is necessarily continuous since the center of $M(G)$ is a semisimple commutative Banach algebra (c.f., [10], Theorem 2.5.17). Since $\|c \cdot a\|_{1}=\|\Psi(c) * a\|_{1} \leqq\|\Psi\|\|c\|\|a\|_{1}$ for all $c \in C, a \in L^{1}(G)$, we have $\|\Psi\| \geqq \chi$. To prove the reverse inequality, first recall that in (1) of the proof $\left\|T_{c}\right\|=\|\Psi c\|$. Since $\left\|T_{c} a\right\|_{1}=$ $\|c \cdot a\|_{1} \leqq \chi\|c\|\|a\|_{1}$, we have $\|\Psi c\| \leqq \chi\|c\|$. Thus, $\|\Psi\| \leqq \chi$ and it follows that $\|\Psi\|=\chi$. Finally, the uniqueness of $\Psi$ is clear since $c \cdot a=\Psi(c) * a=\Phi(c) * a$ for all $c \in C, a \in L^{1}(G)$, implies that

$$
a \rightarrow(\Psi c-\Phi c) * a
$$

is the zero operator on $L^{1}(G)$ and hence has zero norm, which is $\|\Psi c-\Phi c\|$ (by Wendel [3]). Thus $\Psi=\Phi$.

(4) If $C$ is commutative and $G$ is abelian, then for each $c \in C$, $a \in L^{1}(G), \hat{c} \circ \mu \hat{\alpha}=(c \circ a)^{\wedge}=(\Psi(c) * a)^{\wedge}=\left(\Psi_{c}\right)^{\wedge} \hat{a}$. Therefore, $\hat{c} \circ \mu=(\Psi c)^{\wedge}$, proving (iii). The proof is complete.

REMARK. With a few minor modifications in the above proof, we can weaken the hypotheses to obtain roughly the same conclusion. 
Namely, suppose $L^{1}(G)$ is a left $L^{1}(K)$-algebra in the sense that $L^{1}(G)$ is a left $C$-module satisfying the additional associativity condition $c \cdot\left(a * a^{\prime}\right)=(c \cdot a) * a^{\prime}$ for all $c \in C$ and $a \in L^{1}(G)$. Then it can be shown that there is a unique complex homomorphism $\Psi: C \rightarrow M(G)$ such that $c \cdot a=\Psi(c) * a$ for all $c \in C, a \in L^{1}(G) ; \Psi$ is continuous if and only if the module action is continuous, in which case $\|\Psi\|=\chi$. The proof is similar to the above proof. However, the continuity of the linear operator $T_{c}$ reqires a slightly more refined argument. We show that if $\lim _{n-\infty} a_{n}=0$, then $\lim _{n \rightarrow \infty} T_{c}\left(a_{n}\right)=0$. By Hewitt's factorization theorem $([6],(32.23))$ there is a sequence $\left(b_{n}\right)$ in $L^{1}(G)$ and an $a \in L^{1}(G)$ such that $\lim _{n \rightarrow \infty} b_{n}=0$ and $a_{n}=a * b_{n}, n=1,2, \ldots$. It follows that $\lim _{n \rightarrow \infty} T_{c}\left(a * b_{n}\right)=\lim _{n \rightarrow \infty} T_{c}\left(a * b_{n}\right)=\lim _{n-\infty} T_{c}(a) * b_{n}=0$.

3. Application of Cohen's theory of homomorphisms of group algebras. It is easy to see that describing the algebra actions of $L^{1}(K)$ on $L^{1}(G)$ for $L C A$ groups $K$ and $G$ is equivalent to suitably identifying the adjoint maps $\mu: \hat{G} \rightarrow \hat{K}^{0}$. By Theorem 2.5 if $L^{1}(G)$ is an $L^{1}(K)$-algebra there is a continuous homomorphism $\Psi: L^{1}(K) \rightarrow M(G)$ such that $[\Psi c]^{\wedge}=\hat{c} \circ \mu$ for all $c \in L^{1}(K)$, where $\mu: \hat{G} \rightarrow \hat{K}^{0}$ is the adjoint map of the action. At this point the Cohen theory [2] of homomorphisms of commutative group algebras applies. Specific references to the Cohen theory will be taken from the treatment in Rudin's book [12], Chapter 4.

In general, if $\Psi$ is a complex homomorphism of $L^{1}(K)$ into $M(G)$, then $\Psi$ induces a continuous map $\mu: \hat{G} \rightarrow \hat{K}^{0}$ such that $\hat{\Psi} c=\hat{c} \circ \mu$ for all $c \in L^{1}(K)$. Cohen characterizes all homomorphisms $\Psi$ by identifying the respective adjoint maps $\mu$. We turn this around to give an explicit description of $\Psi$ in terms of its action on $L^{1}(K)$.

Before presenting the alledged description of $\Psi$ we set down a few facts and notations. If $H$ is a compact subgroup of the $L C A$ group $G$, then $\pi_{H}$ will denote the canonical homomorphism of $G$ onto $G / H$. Choose a Haar measure on $G / H$ so that the Haar measures of $G, H$, and $G / H$ are canonically related [9] and so that the Haar measure on $H$ is normalized. Let $\mathrm{T}_{H}$ denote the canonical norm decreasing homomorphism of $L^{1}(G)$ onto $L^{1}(G / H)$ given by

$$
T_{H} a(g / H)=\int_{G / H} a(g+h) d h \quad(g / H \in G / H),
$$

for all $a \in L^{1}(G)([6, \mathrm{p} .91]$, or $[9, \mathrm{p} .59])$. Note that by the compactness of $H, T_{H}$ maps $C_{0}(G)$ onto $C_{0}(G / H)$ and in particular the unit ball of $C_{0}(G)$ onto the unit ball of $C_{0}(G / H)$. This fact can be used to show that $\pi_{H}$ induces an isometric isomorphism $\pi_{H}^{*}$ of $M(G / H)$ into $M(G)$ such that

$$
\left\langle f, \pi_{H}^{*} \nu\right\rangle=\left\langle T_{H} f, \nu\right\rangle
$$


for all $f \in C_{0}(G)$ and $\nu \in M(G / H)$. (The mapping $f \rightarrow\left\langle T_{H} f, \nu\right\rangle$ defines a bounded linear functional on $C_{0}(G)$ and hence there is a unique measure $\pi_{H}^{*} \nu \in M(G)$ such that $\left\langle f, \pi_{H}^{*} \nu\right\rangle=\left\langle T_{H} f, \nu\right\rangle$ for all $f \in C_{0}(G)$ ).

We now present our interpretation of the theorem of Paul Cohen [2].

THEOREM 3.1. Let $K$ and $G$ be locally compact Abelian groups. Suppose $\Psi$ is a nonzero complex homomorphism of $L^{1}(K)$ into $M(G)$. Then there are

(i) compact subgroups $H_{1}, \cdots, H_{n}$ in $G$,

(ii) continuous homomorphisms $\theta_{1}, \cdots, \theta_{n}\left(\theta_{i}: K \rightarrow G / H_{i}\right)$,

(iii) pairwise orthogonal nonzero idempotents $e_{1}, \cdots, e_{n}$ in $M(G)$,

(iv) elements $\gamma_{1}, \cdots, \gamma_{n}$ in $\hat{K}$ and $\alpha_{1}, \cdots, \alpha_{n}$ in $\hat{G}$, such that

$$
\Psi(c)=\sum_{i=1}^{n} \alpha_{i} \pi_{H_{i}}^{*} \theta_{i}^{*}\left(\gamma_{i} c\right) * e_{i}
$$

for all $c \in L^{1}(K)$, where $\cdot \pi_{H_{i}}^{*}: M\left(G / H_{i}\right) \rightarrow M(G)$ and $\theta_{i}^{*}: L^{1}(K) \rightarrow M\left(G / H_{i}\right)$ are the canonical maps.

If $\|\Psi\| \leqq 1$, then $\left(^{*}\right)$ simplifies to

$$
\Psi(c)=\alpha \pi_{H}^{*} \theta^{*}(\gamma c)
$$

for all $c \in L^{1}(K)$ and the compact subgroup $H$ and continuous homomorphism $\theta: K \rightarrow G / H$ are uniquely determined.

Proof. Let $\mu: \hat{G} \rightarrow \hat{K}^{0}$ be the adjoint map of $\Psi$. Then by Cohen $\mu$ is a piecewise affine map of $Y \equiv \mu^{-1}(\hat{K}) \subset \widehat{G}$ into $\hat{K}$ ([12], p.78). More explicitly, $Y$ is in the open coset ring of $\hat{G}$ and there are (1) pairwise disjoint sets $S_{1}, \cdots, S_{n}$ in the open coset ring of $\hat{G}$; (2) open cosets $C_{i}$ in $\hat{G}$ such that $S_{i} \subset C_{i}$; (3) for each $i$, affine maps $\mu_{i}$ of $C_{i}$ into $\hat{K}$, such that $\mu$ is the map of $Y=S_{1} \cup \cdots \cup S_{n}$ into $\hat{K}$ which coincides on $S_{i}$ with $\mu_{i}$. Arbitrarily choose $\alpha_{i} \in C_{i}, i=1,2, \cdots, n$, but once chosen we keep $\alpha_{i}$ fixed throughout. Now, for each $i$ set $\gamma_{i}=-\mu_{i}\left(\alpha_{i}\right)\left(=\overline{\mu_{i}\left(\alpha_{i}\right)} \in \hat{K}\right)$. Since $\mu_{i}$ is affine on $C_{i}$ to $\hat{K}$, the map

$$
C_{i}-\alpha_{i} \ni \alpha \rightarrow \mu_{i}\left(\alpha+\alpha_{i}\right)-\mu_{i}\left(\alpha_{i}\right) \in \hat{K}
$$

is a continuous homomorphism of the open subgroup $Q_{i}=C_{i}-\alpha_{i}$ into $\hat{K}$ (and moreover is independent of the choice of $\alpha_{i} \in C_{i}$ ). Let $H_{i}$ be the closed subgroup in $G$ and annihilator of $Q_{i} \subseteq \widehat{G}: H_{i}=Q_{i}^{\perp}$ ([12], p.35). It is well known ([12], Theorem 2.12) by duality theory that the dual group of $G / H_{i}$ can be identified with $H_{i}=Q_{i}$ : if $\beta \in\left(G / H_{i}\right)^{\wedge}$, then $\beta \leftrightarrow \alpha \in Q_{i}$ in such a way that $(g / H, \beta)=(g, \alpha)$ for $g / H \in G / H$. Since $Q_{i}$ is an open subgroup, $H_{i}$ is a compact subgroup in $G$. Using the identification, $\left(G / H_{i}\right)^{\wedge}=Q_{i}$, let $\theta_{i}: K \rightarrow G / H_{i}$ be the 
continuous homomorphism and dual of the continuous homomorphism defined in $(* *)$. The ingredients listed in (i), (ii) and (iv) are now defined. Since $S_{i}$ is a member of the open coset ring in $\hat{G}$, by Cohen [1] the characteristic function of $S_{i}$ is the Fourier-Stieltjes transform of a (nonzero) idempotent measure $e_{i}$ in $M(G)$. Since the $\mathrm{S}_{i}$ are pairwise disjoint, the idempotents $e_{i}$ are pairwise orthogonal, and the ingredients in (iii) are defined.

We need only show that $\Psi(c)$ has the desired representation for each $c \in L^{1}(K)$. Let $\Phi(c)$ denote the element in $M(G)$ given by the right hand side of the equality in $\left(^{*}\right)$. Let $\alpha \in \widehat{G}$. If $\alpha \in \hat{G} \backslash Y$, then since $Y=\mu^{-1}(\hat{K}),(\Psi(c))^{\wedge}(\alpha)=0$, and, since $\operatorname{supp}\left(\hat{e}_{i}\right)=S_{i}$ and $\cup S_{i}=Y$, we have $(\Phi(c))^{\wedge}(\alpha)=0$. If $\alpha \in S_{i} \subset Y$, then since $S_{i} \cap S_{j}=\phi, i \neq j$, and since $\hat{e}_{i}=\chi_{s_{i}}$,

$$
\begin{aligned}
(\Phi(c))^{\wedge}(\alpha) & =\left(\alpha_{i} \pi_{H_{i}}^{*} \theta_{i}^{*}\left(\gamma_{i} c\right)\right)^{\wedge}(\alpha) \\
& =\left(\pi_{H_{i}}^{*} \theta_{i}^{*}\left(\gamma_{i} c\right)\right)^{\wedge}\left(\alpha-\alpha_{i}\right) \\
& =\left(\theta_{i}^{*}\left(\gamma_{i} c\right)\right)^{\wedge}\left(\alpha-\alpha_{i}\right) \quad\left(\text { since } \alpha-\alpha_{i} \in Q_{i}\right) \\
& =\left(\gamma_{i} c\right)^{\wedge}\left(\hat{\theta}_{i}\left(\alpha-\alpha_{i}\right)\right) \\
& =\hat{c}\left(\hat{\theta}_{i}\left(\alpha-\alpha_{i}\right)-\gamma_{i}\right)=\hat{c}\left(\mu_{i}(\alpha)\right) \\
& =\hat{c}(\mu(\alpha))=(\Psi(c))^{\wedge}(\alpha) .
\end{aligned}
$$

Since $\cup S_{i}=Y$, we have $(\Psi(c))^{\wedge}=(\Phi(c))^{\wedge}$ on $G^{\wedge}$ and by the uniqueness of the Fourier-Stieltjes transform, $\Psi(c)=\Phi(c)$ for all $c \in C$.

Finally, if $\Psi$ is norm decreasing then again by Cohen

$$
Y=\mu^{-1}(\hat{K}) \subset \hat{G}
$$

is an open coset and $\mu$ is affine on $Y$ to $\hat{K}$ (c.f., [12], 4.6.3(b), p.88). Choose $\alpha \in Y$, and set $\gamma=-\mu(\alpha)$. Let $H$ be the compact subgroup of $G$ and annihilator of the open subgroup $Y-\alpha$ in $\hat{G}$. Let $\theta$ : $K \rightarrow G / H$ be the dual homomorphism of the continuous homomorphism

$$
(G / H)^{\wedge}=Y-\alpha \ni \alpha^{\prime} \rightarrow \mu\left(\alpha^{\prime}+\alpha\right)-\mu(\alpha) \in \hat{K} .
$$

Then with $\gamma, \alpha, H$ and $\theta$ as defined, it can be shown (as above by taking Fourier-Stieltjes transforms) that $\Psi c=\alpha \pi_{H}^{*} \theta^{*}(\gamma c)$ for all $c \in C$. The uniqueness of $H$ and $\theta$ can be easily verified.

Theorems 2.2 and 3.1 provide the key to the characterization of all nondegenerate algebra actions of $L^{1}(K)$ on $L^{1}(G)$.

Corollary 3.2. Let $K$ and $G$ be locally compact Abelian groups. If $L^{1}(G)$ is a nondegenrate $L^{1}(K)$-algebra then there are compact subgroups $H_{i}$, continuous homomorphisms $\theta_{i}: K \rightarrow G / H_{i}$, pairwise orthogonal nonzero idempotents $e_{i}$ in $M(G)$, and elements $\alpha_{i} \in \hat{G}, \gamma_{i} \in \hat{K}$, $i=1, \cdots, n$, such that 


$$
c \cdot a=\sum_{i=1}^{m} \alpha_{i} \pi_{H_{i}}^{*}\left(\gamma_{i} c *_{\theta_{i}} T_{H_{i}}\left(\bar{\alpha}_{i} a\right)\right) * e_{i}
$$

for all $c \in L^{1}(K), \quad a \in L^{1}(G)$, where $\pi_{H_{i}}^{*}: M\left(G / H_{i}\right) \rightarrow M(G)$ and $T_{H_{i}}$ : $L^{1}(G) \rightarrow L^{1}\left(G / H_{i}\right)$ are the canonical maps, and, where $*_{\theta_{i}}$ is the algebra action of $L^{1}(K)$ on $L^{1}\left(G / H_{i}\right)$ induced by $\theta_{i}$.

If the action of $L^{1}(K)$ on $L^{1}(G)$ is isometric $(\chi \leqq 1)$, then

$$
c \cdot a=\alpha \pi_{H}^{*}\left(\gamma c *_{\theta} T_{H}(\bar{\alpha} \alpha)\right)
$$

for all $c \in L^{1}(K), a \in L^{1}(G)$, and the compact subgroup $H$ and the continuous homomorphism $\theta: K \rightarrow G / H$ are uniquely determined.

Proof. By Theorem 2.2 there is a homomorphism $\Psi$ of $L^{1}(K)$ into $M(G)$ such that $c \cdot a=\Psi c * a, c \in L^{1}(K), a \in L^{1}(G)$, and $\|\Psi\|=\chi$. Now $\Psi$ has the form described in Theorem 3.1. It need only be observed that

$$
\alpha_{i} \pi_{H_{i}}^{*}\left(\gamma_{i} c *_{\theta_{i}} T_{H_{i}}\left(\bar{\alpha}_{i} a\right)\right)=\alpha_{i} \pi_{H_{i}}^{*} \theta_{i}^{*}\left(\gamma_{i} c\right) * a
$$

to complete the proof.

We broaden our notation slightly for the following two corollaries. Namely, if $\gamma \in \hat{K}$ and $\theta \in \operatorname{Hom}(K, G)$, then $\underset{\gamma, \theta}{*}$ will denote the isometric Banach $L^{1}(K)$-algebra action on $L^{1}(G)$ given by

$$
c_{\gamma, \theta}^{*} a(g)=\gamma c *_{\theta} a(g)=\int_{K} \gamma(k) c(k) a(g-\theta(k)) d k .
$$

CoROLLARY 3.3. The following are equivalent for a LCA group $G$.

(i) $\hat{G}$ is connected.

(ii) For every $L C A$ group $K$ and each nondegenerate $L^{1}(K)$ algebra action on $L^{1}(G)$ there is a continuous homomorphism $\theta: K \rightarrow G$ and $\gamma \in \hat{K}$ such that $c \cdot a=c \underset{\gamma, \theta}{*} a$ for all $c \in L^{1}(K)$ and $a \in L^{1}(G)$.

Proof. Now, $G$ has no nontrivial compact subgroups if and only if $\hat{G}$ is connected [5, (24.19)] which implies the open coset ring $\hat{G}$ is $\{\hat{G}, \phi\}$. Thus if $\widehat{G}$ is connected, $H=\{0\}$ in Corollary 3.2, and $\mu^{-1}(\hat{K})=G$; hence we can take $\alpha=1, \gamma=\overline{\mu(1)} . \quad \pi_{\{0\}}$ and $T_{\{0\}}$ reduce to the identity maps on $L^{1}(G)$. Thus (i) implies (ii). If $\widehat{G}$ is not connected, then by choosing a nontrivial compact subgroup $H$ in $G$ and setting $K=G / H$ and $\iota=$ identity on $G / H$, the action $c \cdot a \equiv$ $\pi_{H}^{*}\left(c *, T_{H}(a)\right)$ is not induced by a $\theta \in \operatorname{Hom}(K, G)$ and a $\gamma \in \hat{K}$.

Corollary. 3.4. Let $K$ and $G$ be LCA groups. Suppose $L^{1}(G)$ is a nondegererate $L^{1}(K)$-algebra, $\chi$ is the norm of the action, and $\mu: \hat{G} \rightarrow \widehat{K}^{0}$ is the adjoint map. The following are equivalent. 
(i) There is a (unique) continuous homomorphism $\theta: K \rightarrow G$, and, $\gamma \in \hat{K}$ such that $c \cdot a=c_{\gamma, \theta}^{*} a$ for all $c \in L^{1}(K)$ and $a \in L^{1}(G)$.

(ii) $\hat{G}=\mu^{-1}(\hat{K})$ and $\chi \leqq 1$.

(iii) The linear span of $L^{1}(K) \cdot L^{1}(G)$ is dense in $L^{1}(G)$ and $\chi \leqq 1$.

Proof. (i) $\Rightarrow$ (ii) is clear since $\mu=\hat{\theta}-\gamma$. Conversely, if (ii) holds, then since $\chi \leqq 1, \mu$ is an affine map of $\mu^{-1}(\hat{K})=\hat{G}$ into $\hat{K}$. Set $\gamma=-\mu(1), \theta=(\mu-\gamma)^{\wedge}$, and (i) follows. Now (i) $\Rightarrow$ (iii) since an approximate identity for $L^{1}(K)$ can be shown to be an approximate identity for $L^{1}(G)$. Finally, (iii) $\Rightarrow$ (i) by Hewitt's factorization theorem ([6), Theorem 32.22). Specifically, given any $\alpha \in \widehat{G}$, choose an $\alpha \in L^{1}(G)$ such that $\hat{a}(\alpha) \neq 0$. By the hypothesis in (iii), the factorization theorem implies there is a $c \in L^{1}(K)$ and $a^{\prime} \in L^{1}(G)$ such that $a=c \cdot a^{\prime}$. Then

$$
0 \neq \hat{a}(\alpha)=\hat{c}(\mu(\alpha)) \hat{a}^{\prime}(\alpha),
$$

and hence $\hat{c}(\mu(\alpha)) \neq 0$ and therefore $\mu(\alpha) \in \hat{K}$. Since $\alpha \in \hat{G}$ was arbitrary, $\mu(\hat{G}) \subset \widehat{K}$, i.e., $\mu^{-1}(\hat{K})=\widehat{G}$, and (ii) follows, and therefore (i).

REMARK 3.5. If $G$ is a $L C$ group and if $C$ is a Banach algebra, then every algebra action of $C$ on $M(G)$ is induced by continuous central homomorphism $\Psi: C \rightarrow M(G)$. Clearly, for each $c \in C$, we need only define $\Psi(c)=c \cdot \delta$, where $\delta$ is the identity of $M(G)$; the continuity of $\Psi$ follows as before. Therefore, any separate characterization of the algebra actions of $L^{1}(K)$ on $M(G)$ for $L C A$ groups $K$ and $G$ is unnecessary. Analogues of Corollaries 3.2-3.4 are easily formulated by merely replacing " $L^{1}(G)$ " by " $M(G)$ " in their statements.

REMARK 3.6. In a forthcoming paper [14] we apply the characterization of algebra actions of group algebras obtained in Corollary 3.2 to yield results to topological tensor products of group algebras.

In [11] Rieffel has discussed the tensor product of Banach spaces that are Banach modules over a Banach algebra: if $A$ and $B$ are Banach $C$-modules for a Banach algebra $C$, then the $C$-tensor product of $A$ and $B, A \otimes_{C} B$, is defined as the Banach space and quotient $A \otimes_{r} B / J$, where $J$ is the closed linear subspace generated in the projective tensor product $A \otimes_{\gamma} B$ by all elements of the form

$$
a \cdot c \otimes b-a \otimes c \cdot b, a \in A, b \in B, c \in C .
$$

When $A$ and $B$ are commutative Banach $C$-algebras for a commutative Banach algebra $C$, then $A \otimes_{C} B$ is naturally a commutative Banach $C$-algebra.

We briefly mention two main results obtained in [14]. 
(1) The commutative Banach algebra $L^{1}(G) \otimes_{L^{1}(K)} L^{1}(H)$ is strongly semisimple in all instances of algebra actions of $L^{1}(K)$ on $L^{1}(G)$ and $L^{1}(H)$ for $L C A$ groups $G, H$, and $K$. This can be viewed as a generalization of the work of Gelbaum [3, Theorems 1, §3, §4] and Natzitz [8].

(2) Suppose $L^{1}(G)$ and $L^{1}(H)$ are $L^{1}(K)$-algebras for $L C A$ groups $G, H$, and $K$ and assume the actions are isometric. Let

$$
D=L^{1}(G) \bigotimes_{L^{1}(K)} L^{1}(H),
$$

the commutative Banach $L^{1}(K)$-algebra and $L^{1}(K)$-tensor product of of $L^{1}(G)$ and $L^{1}(H)$. There are unique closed $L^{1}(K)$-ideals $N$ and $\boldsymbol{E}$ in $D$ such that

$$
D=N \oplus \boldsymbol{E}
$$

where $\boldsymbol{E}$ is the essential part [11, Def. 3.5] of $D$ and $L^{1}(K) \cdot \boldsymbol{N}=\{0\}$. Furthermore, there is a canonical $L C A$ group $G \otimes_{K} H$ and an isometric $L^{1}(K)$-isomorphism

$$
\boldsymbol{E}=\left(L^{1}(G) \otimes_{L^{1}(K)} L^{1}(H)\right)_{e} \cong L^{1}\left(G \otimes_{K} H\right) .
$$

Finally, there are compact subgroups $g \subseteq G$ and $h \subseteq H$ such that if $I_{g}$ and $I_{h}$ denote the kernels in $L^{1}(G)$ and $L^{1}(H)$, respectively, of the canonical homomorphisms $T_{g}: L^{1}(G) \rightarrow L^{1}(G / g)$ and $T_{h}: L^{1}(H) \rightarrow L^{1}(H / h)$, then there is a continuous isomorphism

$$
N \cong I_{g} \otimes_{r} I_{h},
$$

where $l_{g} \otimes_{r} l_{h}$ is the projective tensor product of $l_{g}$ and $l_{h}$.

The result above generalizes the work of Gelbaum [3], [4], and Natzitz [8]. Furthermore, using Corollary 3.21 [11], we can obtain a characterization of the space of $L^{1}(K)$-homomorphisms (or multipliers) of $L^{1}(G)$ into $L^{\infty}(H)$. For example, in the special case when $L^{1}(G)$ is an essential $L^{1}(K)$-algebra (and therefore $E=D$ in 2) above), we have

$$
\operatorname{Hom}_{L^{1}(K)}\left(L^{1}(G), L^{\infty}(H)\right) \cong L^{\infty}\left(G \bigotimes_{K} H\right),
$$

where the isomorphism is algebraic and isometric.

\section{REFERENCES}

1. P. J. Cohen, On a conjecture of Littlewood and idempotent measures, Amer. J. Math., 82 (1960), 191-212.

2. - On homomorphisms of group algebras, Amer. J. Math., 82 (1960), 213-226.

3. B. R. Gelboum, Tensor products over Banach algebras, Trans. Amer. Math. Soc., 118 (1965), 131-149.

4. - Tensor products of group algebras, Pacific J. Math., 22 (1967), 241-250. 
5. Edwin Hewitt and K. A. Ross, Abstract harmonic analysis, Vol. 1. Springer-Verlag, Berlin, 1963.

6. — Abstract harmonic analysis, Vol. 2, Springer-Verlag, Berlin, 1970.

7. C. N. Kellogg, Centralizers and $H^{*}$-algebras, Pacific J. Math., 17 (1966), 121-129.

8. Boaz Natzitz, Tensor Products of Banach algebras, Canad. Math. Bull, 11 (1968), 691-701.

9. H. Reiter, Classical harmonic analysis and locally compact groups, Oxford Univ. Press, Oxford, 1968.

10. C. Rickart, General theory of Banach algebras, Van Nostrand, New York, 1960.

11. M. A Rieffel, Induced representations of Banach algebras and locally compact groups, J. Functional Anal., 1 (1967), 443-491.

12. W. Rudin, Fourier analysis on groups, Interscience, New York, 1967.

13. J. G. Wendel, Left centralizers and isomorphisms of group algebras, Pacific J. Math., 2 (1952), 251-261.

14. J. Edward Kerlin, Tensor products of group algebras, (to appear).

Received September 28, 1970.

UNIVERSITY OF KENTUCKY 


\title{
PACIFIC JOURNAL OF MATHEMATICS
}

\author{
EDITORS
}

\author{
H. Samelson \\ Stanford University \\ Stanford, California 94305 \\ C. R. HOBBY \\ University of Washington \\ Seattle, Washington 98105
}

J. DugundJI

Department of Mathematics

University of Southern California

Los Angeles, California 90007

RICHARD ARENS

University of California

Los Angeles, California 90024

\section{ASSOCIATE EDITORS}

E. F. BECKENBACH
B. H. NEUMANN

F. WoLF
K. YosHIDA

\section{SUPPORTING INSTITUTIONS}

\author{
UNIVERSITY OF BRITISH COLUMBIA \\ CALIFORNIA INSTITUTE OF TECHNOLOGY \\ UNIVERSITY OF CALIFORNIA \\ MONTANA STATE UNIVERSITY \\ UNIVERSITY OF NEVADA \\ NEW MEXICO STATE UNIVERSITY \\ OREGON STATE UNIVERSITY \\ UNIVERSITY OF OREGON \\ OSAKA UNIVERSITY \\ UNIVERSITY OF SOUTHERN CALIFORNIA
}

\author{
STANFORD UNIVERSITY \\ UNIVERSITY OF TOKYO \\ UNIVERSITY OF UTAH \\ WASHINGTON STATE UNIVERSITY \\ UNIVERSITY OF WASHINGTON \\ $\stackrel{*}{*} \stackrel{*}{*} \stackrel{*}{*}{ }^{*}{ }^{*}$ MERICAN MATHEMATICAL SOCIETY \\ CHEVRON RESEARCH CORPORATION \\ NAVAL WEAPONS CENTER
}

The Supporting Institutions listed above contribute to the cost of publication of this Journal, but they are not owners or publishers and have no responsibility for its content or policies.

Mathematical papers intended for publication in the Pacific Journal of Mathematics should be in typed form or offset-reproduced, (not dittoed), double spaced with large margins. Underline Greek letters in red, German in green, and script in blue. The first paragraph or two must be capable of being used separately as a synopsis of the entire paper. The editorial "we" must not be used in the synopsis, and items of the bibliography should not be cited there unless absolutely necessary, in which case they must be identified by author and Journal, rather than by item number. Manuscripts, in duplicate if possible, may be sent to any one of the four editors. Please classify according to the scheme of Math. Rev. Index to Vol. 39. All other communications to the editors should be addressed to the managing editor, Richard Arens, University of California, Los Angeles, California, 90024.

50 reprints are provided free for each article; additional copies may be obtained at cost in multiples of 50 .

The Pacific Journal of Mathematics is published monthly. Effective with Volume 16 the price per volume (3 numbers) is $\$ 8.00$; single issues, $\$ 3.00$. Special price for current issues to individual faculty members of supporting institutions and to individual members of the American Mathematical Society: $\$ 4.00$ per volume; single issues $\$ 1.50$. Back numbers are available.

Subscriptions, orders for back numbers, and changes of address should be sent to Pacific Journal of Mathematics, 103 Highland Boulevard, Berkeley, California, 94708.

PUBLISHED BY PACIFIC JOURNAL OF MATHEMATICS, A NON-PROFIT CORPORATION

Printed at Kokusai Bunken Insatsusha (International Academic Printing Co., Ltd.), 7-17, Fujimi 2-chome, Chiyoda-ku, Tokyo, Japan. 


\section{Pacific Journal of Mathematics}

J. T. Borrego, Haskell Cohen and Esmond Ernest Devun, Uniquely

representable semigroups on the two-cell .................. 565

Glen Eugene Bredon, Some examples for the fixed point property ........ 571

William Lee Bynum, Characterizations of uniform convexity .......... 577

Douglas Derry, The convex hulls of the vertices of a polygon of order $n \ldots 583$

Edwin Duda and Jack Warren Smith, Reflexive open mappings .......... 597

Y. K. Feng and M. V. Subba Rao, On the density of $(k, r)$ integers ........ 613

Irving Leonard Glicksberg and Ingemar Wik, Multipliers of quotients of

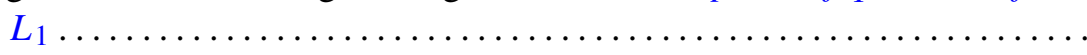

John William Green, Separating certain plane-like spaces by Peano

continua.........................................

Lawrence Albert Harris, A continuous form of Schwarz's lemma in normed

linear spaces .................................... 635

Richard Earl Hodel, Moore spaces and $w$-spaces ............... 641

Lawrence Stanislaus Husch, Jr., Homotopy groups of PL-embedding spaces.

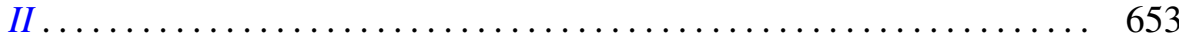

Yoshinori Isomichi, New concepts in the theory of topological

space-supercondensed set, subcondensed set, and condensed set.....

J. E. Kerlin, On algebra actions on a group algebra .................

669

Keizō Kikuchi, Canonical domains and their geometry in $C^{n} \ldots \ldots \ldots \ldots 681$

Ralph David McWilliams, On iterated $w^{*}$-sequential closure of cones. .

697

C. Robert Miers, Lie homomorphisms of operator algebras ..

717

Louise Elizabeth Moser, Elementary surgery along a torus knot ...

737

Hiroshi Onose, Oscillatory properties of solutions of even order differential

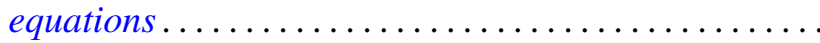

747

Wellington Ham Ow, Wiener's compactification and $\Phi$-bounded harmonic

functions in the classification of harmonic spaces...

Zalman Rubinstein, On the multivalence of a class of meromorphic

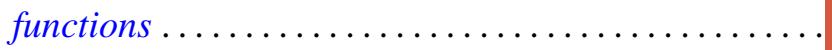

771

785

Hans H. Storrer, Rational extensions of modules

795

topological lattices ...........................

Robert Evert Stong, On the cobordism of pairs .................. 803
Albert Leon Whiteman, An infinite family of skew Hadamard matrices . . . 817

Lynn Roy Williams, Generalized Hausdorff-Young inequalities and mixed

norm spaces 